\title{
A Pseudoaneurysm of the Jugular Vein: A Case Report in a 74
}

\section{Years Old Patient}

\section{Sanin-Pérez E ${ }^{1}$, Gil-Serrano $\mathrm{PE}^{2 *}$, Andres $\mathrm{YV}^{3}$ and Chaustre-}

Soledad JE4

1Interventional Radiologist, Hospital Pablo Tobon Uribe, Colombia

${ }^{2}$ Medical Radiologist, CES University, Colombia

${ }^{3}$ Resident Physician of Radiology, Universidad De la Sabana, Colombia

${ }^{4} \mathrm{MD}$ intern, Universidad De la Sabana, Colombia

*Corresponding author: Patricia Gil-Serrano, Medical Radiologist, CES University, Cali, Colombia, Tel: +57 (318) 529 -

8676; Email: patricialgil16@hotmail.com

\section{Abstract}

The venous pseudoaneurysms of the neck are uncommon clinical entities that usually involve the internal jugular vein and less frequently the external jugular vein. Very few cases have been reported in the worlds academic medical literature. The pseudoaneurysms of the cervical venous system are infrequent due to the low pressure in the vena cava. The most frequent causes include vein wall injury due to multiple puncture attempts to obtain central venous access, surgery, local damage due to intravenous drug abuse and trauma.

Keywords: Venous Pseudoaneurysms; Jugular Vein; Ultrasound; Venous Aneurysm

Abbreviation: DSA: Digital Subtraction Angiography.

\section{Key Messages}

a) The pseudoaneurysms are generally formed by arterial blood, secondary to the high blood pressure that maintains permeability of the lesion in the blood vessel [1-3].

b) Doppler color and digital subtraction angiography (DSA) are the gold standard used for the diagnosis of the venous pseudoaneurysm in the neck [1].

\section{Introduction}

We presented the case of a 74 year old male patient, with previous medical history of multiple comorbidities that is taken into the emergency room due to deterioration in his functional class that has developed in the past month. This patient had no symptoms suggestive of jugular pseudoaneurisma and went to the emergency department secondary to a decompensated heart failure associated to hypervolemia due to poor ultrafiltration of the peritoneal dialysis and failure of the peritoneal membrane. In whom a preliminary neck ultrasound is performed evidencing an incidental finding of a saccular image that is hypoechoic, with internal flow upon evaluation with Doppler color, located in the right supraclavicular space and is $3 \mathrm{~cm}$ large in its greatest diameter. A conclusion is drawn that the pseudoaneurysm of the jugular vein is a rare complication of a routine procedure, and must be preferably observed by interventional radiologists under echographic guidance. Doppler color and digital subtraction angiography (DSA) are the gold standard used for the diagnosis of the venous pseudoaneurysm of the neck and its treatment may be conservative in the absence of symptoms or complications. 


\section{Clinical Radiology \& Imaging Journal}

\section{Case History}

We report the case of a 74 year old, male patient, with previous medical history of hypertension, chronic kidney disease in terminal stage with peritoneal dialysis, heart failure and pulmonary hypertension. The patient arrives to the emergency room due to deterioration in his functional class that has developed in the past month. Secondary to a decompensated heart failure associated to hypervolemia due to poor ultrafiltration of the peritoneal dialysis and failure of the peritoneal membrane. The patient is hospitalized to optimize treatment of underlying conditions. There was no cyanosis of the fingertips or edema of the fingers. There was no history of tingling and / or numbness in the upper extremities. There was no history of pain in the chest or neck. There was no history of pain even in the hyperabduction of the arms. There was no edema in the right upper extremity or dilated veins on the face. There was no history of ecchymosis or signs of skin discoloration suggesting rupture of the external jugular vein. All this indicated that the pseudo aneurysm had developed slowly and progressively so the patient did not develop severe symptoms. The patient is taken to the interventional radiology department for the placement of a high flow catheter for hemodialysis. In the preliminary ultrasound of the neck vessels, a saccular image is observed; it is hypoechoic, with internal flow upon evaluation with Doppler color, located in the right supraclavicular space and is $3 \mathrm{~cm}$ large in its greatest diameter. Following its visualization it is punctured and contrast medium is injected; which defines it as a venous pseudoaneurysm secondary to a precise lesion located in the jugulo subclavian confluence (Figures $1 \& 2$ ), probably related to multiple puncture attempts to obtain central venous access from previous catheters. The brachiocephalic vein and the superior vena cava were found completely permeable. Upon the absence of symptoms related to the pseudoaneurysm, it was decided not to be intervened. A high flow catheter for hemodialysis was placed without any complications (Figure 3).

Figure 1: Venous angiography: Venous pseudoaneurysm secondary to a precise lesion located in the jugulo-subclavian confluence probably related to multiple puncture attempts to obtain central venous access from previous catheters.
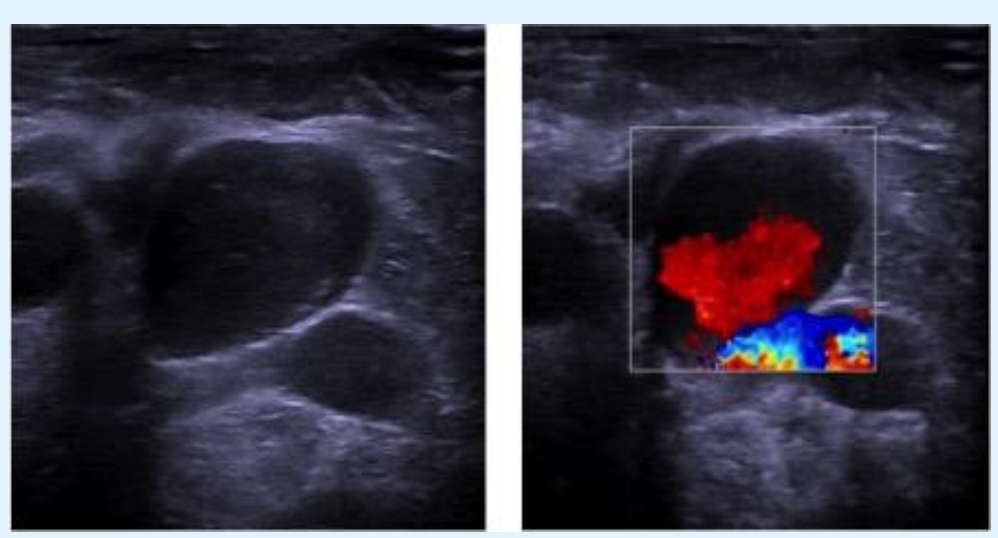

Figure 2: Ultrasound image with finding of jugular vein aneurysm with evidence of internal flow with color Doppler signal. 


\section{Clinical Radiology \& Imaging Journal}

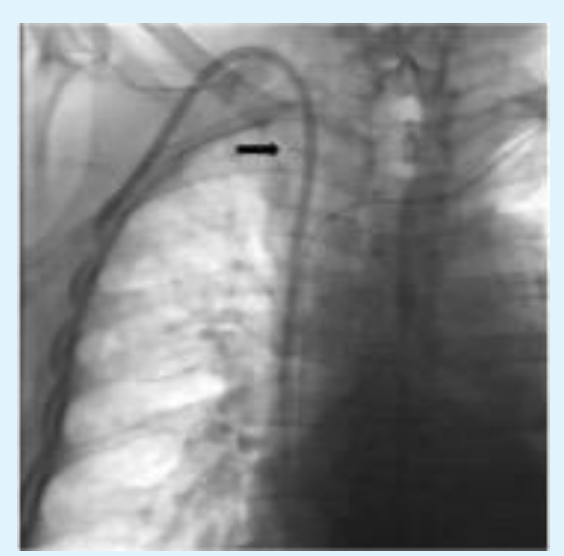

Figure 3: Venous angiography: Post-procedural chest x-ray that evidences a high flow catheter that is adequately placed.

\section{Discussion}

A pseudoaneurysm is defined as a collection of blood that is contained by perivascular soft tissues that form a fibrous capsule around it and generate what may be called a "pulsating hematoma". The pseudoaneurysms are generally formed by arterial blood, secondary to the high blood pressure that maintains permeability of the lesion in the blood vessel. On the contrary, the veins, with their low pressure, generally allow an effective occlusion of the defect in the vessel and thus, venous pseudoaneurysms are much less frequent than arterial ones [3]. A venous pseudoaneurysm after a venipuncture is a rare complication of a routine procedure. Its most common cause is the lesion of the vessel wall induced by several attempts of puncturing the vein to obtain a central venous access [4]. Also, pseudoaneurysms have been described by being surgically induced, similar to those associated to traumatic lesions and intravenous drug abuse [3].

The natural history of these pseudoaneurysms depends on their anatomic location. Those located in the cervical region; generally have a benign course that merely causes pain and tenderness in the area [6]. Nevertheless, some complications include venous thrombosis, pulmonary emboli, thrombophlebitis, compression or distortion of adjacent structures and rarely, rupture [3]. Patients may be asymptomatic, but in some occasions may also refer cervical pulsating masses, and may even be accompanied by a painful edema that is associated with thrombophlebitis $[3,5]$. Doppler color and digital subtraction angiography (DSA) are the gold standard used for the diagnosis of the venous pseudoaneurysm in the neck [1], but these may be adequately characterized by other imaging methods such as contrast tomography or magnetic resonance. The ultrasound allows the ability to observe the presence of an anechoic lesion that is perivascular, with low-speed turbulent blood flow that may be surrounded by a hematoma that has poorly defined borders in acute cases and has well defined borders in chronic cases. With these methods, added to the Doppler color, other vascular lesions may also be identified; such as arterial pseudoaneurysms and arteriovenous fistulas; allowing a more specific and precise diagnosis [6]. The differential diagnosis includes lymph nodes, neoplasms, and hematomas, vascular anomalies such as arteriovenous fistula, arterial aneurysm, cavernous hemangioma, laryngocele, thyroid gland nodules, lipomas, thyroglossal duct cysts and branchial cysts [1]. Mohanty, et al. showed that in elderly patients, hypertensive, compression of the left innominate vein by a high atherosclerotic aorta could contribute to the formation of fusiform dilation of the affected vessel and / or its tributaries [7]. The surgical resection is the recommended treatment in patients with large and symptomatic venous pseudoaneurysms. Other therapeutic options include: observation, compression, percutaneous injection of thrombin and embolization with coils $[2,3]$.

\section{References}

1. Verma RK, Kaushal D, Panda NK (2013) External jugular vein aneurysm with thrombus presenting as painful neck mass: a case report. Oman Medical J 28(4): 278-280.

2. Lotfi M, Nabavizadeh SA, Abbasi HR, Geramizadeh B (2007) Iatrogenic venous pseudoaneurysm following 


\section{Clinical Radiology \& Imaging Journal}

venipuncture: case report and review of the literature. J Clin Ultrasound 35(9): 521-523.

3. Ward WH, Platz TA, Pond KT, Southern FA, Moore EM (2009) Iatrogenic venous pseudoaneurysm: case report and review of the literature. Vascular 17(6): 355-358.

4. Luckraz H (2003) Venous pseudo-aneurysm as a late complication of short-term central venous catheterisation. Cardiovasc Ultrasound 1: 6.

5. Lee HY, Cho SH, Ko TY, Kim HS, Kim JI, et al. (2014) Saccular aneurysm of the external jugular vein: a case report. Korean J Thorac Cardiovasc Surg 47(2): 171173.

6. Drakonaki EE, Symvoulakis EK, Fachouridi A, Kounalakis D, Tsafantakis E (2011) External jugular vein aneurysm presenting as a cervical mass. International journal of otolaryngology 2011: 485293.

7. Mohanty D, Jain BK, Garg PK, Tandon A (2013) External jugular venous aneurysm: A clinical curiosity. J Nat Sci Biol Med 4(1): 223-225.

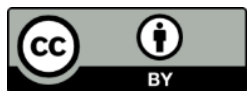

